\title{
HIGHLIGHTS
}

INFECTION

\section{Intravesical hyaluronic acid and chondroitin reduces incidence of recurrent UTI}

Prophylactic instillation of hyaluronic acid (HA) and chondroitin sulfate (CS) directly into the bladder can reduce the rate of urinary tract infection (UTI) in women prone to recurrent episodes, according to a recent report in European Urology.

A team based at the Graeca University of Catanzaro, Italy, investigated the effect of intravesical administration of an HA-CS mixture on UTI rate and quality of life in women over a 12-month period.

In their double-blind, randomized, placebo-controlled trial, 57 women underwent baseline assessment of clinical parameters and quality of life before intravesical instillation of $50 \mathrm{ml}$ of HA-CS solution or placebo (saline) every week for 1 month and monthly for the next 5 months.

At 12 months there was a significant decrease (mean $87 \%$ ) in UTI rate among women in the HA-CS group. At this point (study end), $48 \%$ of women in the HA-CS group were free from recurrence of UTI, whereas all participants in the placebo group had experienced at least one UTI episode. Furthermore, quality of life had improved in the HA-CS group as early as 1 month into the study, with no reports of adverse events.

The interaction between bacteria and the bladder epithelium is a recognized factor in the pathogenesis of UTI. Damage to the epithelial glycosaminoglycan layer is thought to enhance bacterial adherence, increasing the likelihood of infection. HA (a mucopolysaccharide) and CS (which bolsters mucosal integrity) have previously been used to restore the glycosaminoglycan layer in patients with interstitial cystitis. These new data indicate that HA-CS instillation could also be an effective form of UTI prophylaxis.

Although the cost exceeds that of prophylactic antibiotics, the intravesical HA-CS strategy does not exacerbate the problem of antibiotic resistance. Further studies, perhaps comparing the efficacy

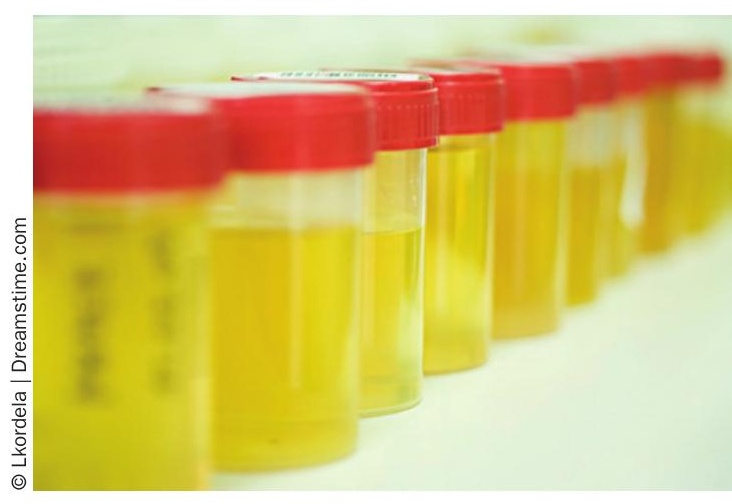

of intravesical HA-CS with that of prophylactic antimicrobials, will clarify the potential clinical benefits of the former.

\section{Annette Fenner}

Original article Damiano, R. et al. Prevention of recurrent urinary tract infections by intravesical administration of hyaluronic acid and chondroitin sulphate: a placebocontrolled randomised trial. Eur. Urol. doi:10.1016/ j.eururo.2010.12.039 